\title{
Case-control study on risk factors associated with Brucella melitensis in goat farms in Peninsular Malaysia
}

\begin{abstract}
Caprine brucellosis is a bacterial zoonotic infection affecting goats especially in developing countries all over the world. In Malaysia, the risk factors associated with this infection in farms have not been studied. A case-control study was carried out in goat farms in four states of Malaysia to elucidate the risk factors associated with the infection on the farms using structured questionnaires and face-to-face interviews. Results indicate that the introduction of new animals $(\mathrm{OR}=5.25 ; 90 \% \mathrm{CI}=1.46,18.88)$, younger age category of farms $(\mathrm{OR}=5.53$; $90 \% \mathrm{CI}=1.09,21.66)$, and farms with single breed of goats $(\mathrm{OR}=8.50 ; 90 \% \mathrm{CI}=1.27$, 41.97) were significant risk factors for brucellosis. In order to control brucellosis or possibly eradicate it in goat farms, these factors need to be dealt with. Enforcing stringent importation protocols or complete ban of goat importation from brucellosis endemic countries will help reduce risk of introducing new infection into the country.
\end{abstract}

Keyword: Case-control; Brucella melitensis; Risk factors; Goats; Farms; Malaysia 